\title{
A REPRESENTATION OF JACOBI FUNCTIONS
}

\author{
E. Y. DEEBA \\ Department of Applied Mathematical Sciences \\ University of Houston-Downtown \\ Houston, Texas 77002 \\ and \\ E. L. KOH \\ Department of Mathematics \& Statistics \\ University of Regina \\ Regina, Canada S45 0A2 \\ (Received August 1, 1985)
}

Abstract: Recently, the continuous Jacobi transform and its inverse are defined and studied in [1] and [2]. In the present work, the transform is used to derive a series representation for the Jacobi functions $P_{\lambda}^{(\alpha, \beta)}(x),-\frac{1}{2} \leq \alpha, \beta \leq \frac{1}{2}, \alpha+\beta=0$, and $\lambda \geq-\frac{1}{2}$. The case $\alpha=\beta=0$ yields a representation for the Legendre functions and has been dealt with in [3]. When $\lambda$ is a positive integer $n$, the representation reduces to a single term, viz., the Jacobi polynomial of degree $n$.

KEY WORDS AND PHRASES: Jacobi functions, Jacobi transform, representation, special functions.

1980 MATHEMATICS SUBJECT CLASSIFICATION CODES: 33A30, 44A15, 44A20.

1. Introduction. The continuous Jacobi transform and its inverse were introduced and studied in [1] and [2]. These transforms generalize the work of Butzer, Stens and Wehrens [3] on the continuous Legendre transform and the work of Debnath [4] on the discrete Jacobl transform. In [2] an application to sampling technique was given. In the present work, the continuous Jacobl transform is used to derıve a series representation of Jacobi functions $P_{\lambda}^{(\alpha, \beta)}(x)$. The representation includes that for the Legendre function given in [3]. When $\lambda$ is a positive integer, the representation reduces to the Jacobi polynomial (see e.g. [5]). 
2. Preliminaries. In this section we review material needed in the development of the paper.

For $\alpha, \beta>-1, \lambda \in R, \lambda+\alpha+\beta \neq 0,-1,-2, \ldots$ and $x \varepsilon(-1,1]$, the Jacobi function of the first kind, ${ }_{\lambda}^{(\alpha, \beta)}(x)$, is given by

$$
\mathrm{P}_{\lambda}^{(\alpha, \beta)}(\mathrm{x})=\frac{\Gamma(\lambda+\alpha+1)}{\Gamma(\lambda+1) \Gamma(\alpha+1)} \mathrm{F}\left(-\lambda, \lambda+\alpha+\beta+1 ; \alpha+1 ; \frac{1-\mathrm{x}}{2}\right)
$$

(see [6]) where

$$
F(a, b ; c ; z)=\sum_{k=0}^{\infty} \frac{(a)_{k}^{(b)} k}{(c)_{k} k !} z^{k},|z|<1,
$$

$a, b, c$ real numbers with $c \neq 0,-1,-2, \ldots$.

$$
\text { Since } \mathrm{p}_{\lambda}^{(\alpha, \beta)}(\mathrm{x})=\frac{\Gamma(\alpha-\lambda+1) \Gamma(\lambda-\alpha-\beta)}{\Gamma(1-\lambda) \Gamma(\lambda-\beta)} \mathrm{P}_{\lambda-\alpha-\beta-1}^{(\alpha, \beta)}(\mathrm{x}) \text {, we may }
$$

restrict ourselves to $\lambda \geq-\frac{\alpha+\beta+1}{2}$. The function $\mathrm{P}_{\lambda}^{(\alpha, \beta)}(\mathrm{x})$ satisfies the following relations:

$$
\begin{aligned}
& \frac{d}{d x}\left(w(x)\left(1-x^{2}\right) \frac{d}{d x} P_{\lambda}^{(\alpha, \beta)}(x)\right)=-\lambda(\lambda+\alpha+\beta+1) w(x) P_{\lambda}^{(\alpha, \beta)}(x) \\
& P_{\lambda}^{(\alpha, \beta)}(1)=\frac{\Gamma(\lambda+\alpha+1)}{\Gamma(\lambda+1) \Gamma(\alpha+1)},
\end{aligned}
$$

and

$$
\begin{aligned}
\left(1-x^{2}\right) \frac{d}{d x} P_{\lambda}^{(\alpha, \beta)}(x) & =\left(\frac{\lambda(\alpha-\beta)}{2 \lambda+\alpha+\beta}-\lambda x\right) P_{\lambda}^{(\alpha, \beta)}(x) \\
& +\frac{2(\lambda+\alpha)(\lambda+\beta)}{2 \lambda+\alpha+\beta} P_{\lambda-1}^{(\alpha, \beta)}(x) .
\end{aligned}
$$

For a proof of (2.2), (2.3) and (2.4) see [1]. The term w(x) in (2.2) is the weight function $w(x)=(1-x)^{\alpha}(1+x)^{\beta}$ and will be used throughout the paper. Furthermore, it was shown in [1] that for $\lambda \geq-\frac{\alpha+\beta+1}{2}$ and for any $x \in(-1,1]$.

$$
\left|P{ }_{\lambda}^{(\alpha, \beta)}(x)\right| \leq \frac{\Gamma(\lambda+\alpha+1)}{\Gamma(\lambda+1) \Gamma(\alpha+1)}+M(\lambda, \alpha, \beta) \log \frac{2}{1+x}
$$

where $M(\lambda, \alpha, \beta)$ is some constant depending upon $\lambda, \alpha$ and $\beta$; and for any $\lambda, v \geq-\frac{\alpha+\beta+1}{2}, \lambda \neq v, \lambda \neq-(v+\alpha+\beta+1), \alpha>-\frac{1}{2},-\frac{1}{2}<\beta<\frac{1}{2}$ we have the relation

$$
\begin{aligned}
& \frac{1}{2^{\alpha+\beta+1}} \int_{-1}^{1} w(x) P_{\lambda}^{(\alpha, \beta)}(x) P_{v}^{(\beta, \alpha)}(-x) d x \\
& \quad=\frac{\Gamma(\lambda+\alpha+1) \Gamma(v+\beta+1)}{\Gamma(\lambda-v)(\lambda+v+\alpha+\beta+1)}\left\{\frac{\sin \pi \lambda}{\Gamma(v+1) \Gamma(\lambda+\alpha+\beta+1)}-\frac{\sin \pi \nu}{\Gamma(\lambda+1) \Gamma(v+\alpha+\beta+1)}\right\} .
\end{aligned}
$$


We shall denote, throughout, the weighted square integrable functions on $(-1,1)$ by $L_{w}^{2}(-1,1)$. For $f \in L_{w}^{2}(-1,1), \alpha>-\frac{1}{2},-\frac{1}{2}<\beta<\frac{1}{2}$, the continuous Jacobi transform (see [1]) is defined by

$$
\hat{f}^{(\alpha, \beta)}(\lambda)=\frac{1}{2^{\alpha+\beta+1}} \int_{-1}^{1} w(x) P_{\lambda}^{(\alpha, \beta)}(x) f(x) d x
$$

When $\alpha=\beta=0, \hat{f}^{(\alpha, \beta)}$ reduces to the continuous Legendre transform studied in [3] and when $\lambda=n \in P$ ( $P$, the set of non-negative integers), $\hat{f}(\alpha, \beta)$ reduces to the discrete Jacobi transform of Debnath [4].

It was shown in [1] that if $\lambda^{\frac{1}{2}} f(\alpha, \beta)\left(\lambda-\frac{1}{2}\right) \in L^{l}\left(R^{+}\right)$and if $\alpha+\beta=0$ then for almost every $x \in(-1,1)$, we obtain the inversion formula where$$
f(x)=4 \int_{0}^{\infty} \hat{f}(\alpha, \beta)\left(\lambda-\frac{1}{2}\right) P_{\lambda-\frac{1}{2}}^{(\alpha, \beta)}(-x) H_{0}(\lambda) \lambda \sin \pi \lambda d \lambda
$$

$$
H_{0}(\lambda)=\frac{\Gamma^{2}\left(\lambda+\frac{1}{2}\right)}{\Gamma\left(\lambda+\alpha+\frac{1}{2}\right) \Gamma\left(\lambda+\beta+\frac{1}{2}\right)} .
$$

Since we needed the condition $\alpha+\beta=0$ to derive (2.8), we shall, from now on, assume this condition on $\alpha$ and $\beta$.

In [2] the second continuous Jacobi transform was studied. For $\lambda^{-\beta+\frac{1}{2}} f \in L^{1}\left(R^{+}\right)$, it is given by

$$
\hat{A}_{f}(\alpha, \beta)(x)=4 \int_{0}^{\infty} f(\lambda) P_{\lambda-\frac{1}{2}}^{(\beta)}(-x) \frac{\Gamma\left(\lambda+\frac{1}{2}\right)}{\Gamma\left(\lambda+\beta+\frac{1}{2}\right)} \lambda \sin \pi \lambda d \lambda
$$

and the associated inversion formula is

$$
f(\lambda)=\frac{1}{2} \frac{\Gamma\left(\lambda+\alpha+\frac{1}{2}\right)}{\Gamma\left(\lambda+\frac{1}{2}\right)} \int_{-1}^{1} w(x) P_{\lambda-\frac{1}{2}}^{(\alpha, \beta)}(x) \wedge f(\alpha, \beta)(x) d x
$$

The relation between the different transforms (see [2]) is

$$
\left(\hat{f}^{(\alpha, \beta)}(\cdot)\right)^{\wedge(\alpha, \beta)}(\lambda)=\frac{2 \Gamma\left(\lambda+\alpha+\frac{1}{2}\right)}{\Gamma\left(\lambda+\frac{1}{2}\right)} f(\lambda)
$$

and

$$
\left(\frac{\Gamma\left(\lambda+\frac{1}{2}\right)}{\Gamma\left(\lambda+\alpha+\frac{1}{2}\right)} \hat{f}^{(\alpha, \beta)}(\cdot)\right)(\alpha, \beta)(x)=f(x) .
$$

As an application of (2.9) and (2.10), it was shown in [2] that if $\mathrm{F} \in \mathrm{C}\left(\mathrm{R}^{+}\right)$is given by

$$
F(\lambda)=\frac{1}{2} \int_{-1}^{1} w(x) f(x) P_{\mu \lambda-\frac{1}{2}}^{(\alpha, \beta)}(x) d x
$$


for some $\mu>0, f \in L_{W}^{2}(-1,1)$, then for all $\lambda \in R^{+}$, we have

$$
F(\lambda)=\sum_{n=0}^{\infty} \frac{(2 n+1) \Gamma(n+1) \Gamma\left(\mu \lambda+\alpha+\frac{1}{2}\right) \sin \pi\left(\lambda \mu-\left(n+\frac{1}{2}\right)\right)}{\pi\left(\lambda^{2} \mu^{2}-\left(n+\frac{1}{2}\right)^{2}\right) \Gamma(n+\alpha+1) \Gamma\left(\lambda \mu+\frac{1}{2}\right)} F\left(\frac{n+\frac{1}{2}}{\mu}\right) .
$$

We will employ $(2.7),(2.8),(2.9)$ and $(2.10)$ to derive the representation formula of the Jacobi functions. Since $\alpha+\beta=0$, we shall write $\mathrm{P}_{\lambda}^{(\alpha, \beta)}(\mathrm{x})$ as $\mathrm{P}_{\lambda}^{(\alpha,-\alpha)}(\mathrm{x})$.

3. Derivation of the Representation Formula. Again, throughout this section we shall assume $\alpha+\beta=0,-\frac{1}{2}<\alpha, \beta<\frac{1}{2}$ and $\alpha \neq 0$. The case $\alpha=0$ reduces to the representation of the Legendre functions and has been developed in [3].

The series representation that we will develop, in this section, for $\mathrm{P}^{(\alpha,-\alpha)}(\mathrm{x})$ is

$$
\begin{aligned}
& P_{\lambda}^{(\alpha,-\alpha)}(x)=\frac{\Gamma(\lambda+\alpha+1)}{\Gamma(\lambda+1) \Gamma(\alpha+1)} \frac{\sin \pi \lambda}{\pi} . \\
& \quad \cdot \lambda(\lambda+1) \sum_{n=1}^{\infty} \frac{(2 n+1) n !(-1)^{n} P_{n}^{(\alpha,-\alpha)}(x)}{n(n+1)(\alpha+1)} n^{(\lambda-n)(\lambda+n+1)}+1 \\
& \left.+\frac{1}{\lambda(\lambda+1)}-\sum_{n=0}^{\infty} \frac{(-1)^{n}}{\alpha+n+1}\left(\frac{1-x}{1+x}\right)^{n+1}\right\}, 0 \leq x<1,
\end{aligned}
$$

and

$$
\begin{aligned}
& P_{\lambda}^{(\alpha,-\alpha)}(x)=\frac{\Gamma(\lambda+\alpha+1)}{\Gamma(\lambda+1) \Gamma(\alpha+1)} \frac{\sin \pi \lambda}{\pi} . \\
& \left\{\lambda(\lambda+1) \sum_{n=1}^{\infty} \frac{(2 n+1) n !(-1)^{n} P_{n}(\alpha,-\alpha)(x)}{n(n+1)(\alpha+1){ }_{n}^{(\lambda-n)(\lambda+n+1)}}+1+\frac{1}{\lambda(\lambda+1)}\right. \\
& -\frac{1}{\alpha}+\left(\frac{1+x}{1-x}\right)^{\alpha} \frac{\pi}{\sin \pi \alpha}+\sum_{n=0}^{\infty} \frac{(-1)^{n}}{\alpha-n-1}\left(\frac{1+x}{1-x}\right)^{n+1},-1<x \leq 0 .
\end{aligned}
$$

In order to derive (3.1) and (3.2), we shall first introduce an auxillary function $k(x ; h)$, apply $(2.7),(2.9)$ to $k(x ; h)$ and utilize the uniqueness of the Jacobi transform.

Lemma 3.1. For he $(-1,1)$, define

$$
k(x ; h)= \begin{cases}\frac{1}{\alpha}\left[\left(\frac{1+x}{1-x}\right)^{\alpha}-\left(\frac{1+h}{1-h}\right)^{\alpha}\right], & h \leq x<1, \\ 0 \quad,-1<x \leq h\end{cases}
$$

Then 


$$
\hat{k}^{(\alpha,-\alpha)}(x ; h)(\lambda)=\begin{aligned}
& \frac{1}{\lambda(\lambda+1)}\left\{\frac{\Gamma(\lambda+\alpha+1)}{\Gamma(\lambda+1 ! \Gamma(\alpha+1)}-p_{\lambda}^{(\alpha,-\alpha)}(h)\right\}, \lambda \neq 0, \lambda \geq-\frac{1}{2} \\
& \frac{J}{2 \alpha}\left\{1-h-\left(\frac{1+h}{1-h}\right)^{\alpha} \int_{h}^{1}\left(\frac{1-x}{1+x}\right) \alpha d x\right\}, \lambda=0 .
\end{aligned}
$$

Proof. (2.2) together with (2.7) yields for $\lambda \neq 0$ and $\alpha+\beta=0$

$$
\begin{aligned}
& \hat{k}^{(\alpha,-\alpha)}(\cdot ; h)(\lambda)=\frac{1}{2} \int_{-1}^{1}(1-x)^{\alpha}(1+x)^{-\alpha} P_{\lambda}(\alpha,-\alpha)(x) k(x ; h) d x \\
& =-\frac{3}{2} \frac{1}{\lambda(\lambda+1)} \int_{-1}^{1} \frac{d}{d x}\left((1-x)^{\alpha+1}(1+x)^{-\alpha+1} \frac{d}{d x} P_{\lambda}^{(\alpha,-\alpha)}(x)\right) k(x ; h) d x .
\end{aligned}
$$

On integrating by parts, we obtain

$$
\hat{\mathrm{k}}^{(\alpha,-\alpha)}(\cdot ; \mathrm{h})(\lambda)=\frac{1}{\lambda(\lambda+1)} \int_{\mathrm{h}}^{1} \frac{\mathrm{d}}{\mathrm{dx}} \mathrm{P}_{\lambda}^{(\alpha,-\alpha)}(\mathrm{x}) \mathrm{dx}
$$

from which it follows that for $\lambda \neq 0, \lambda \geq-\frac{1}{2}$

$$
\hat{k}^{(\alpha,-\alpha)}(\cdot ; h)(\lambda)=\frac{1}{\lambda(\lambda+1)}\left\{P_{\lambda}^{(\alpha,-\alpha)}(1)-P_{\lambda}^{(\alpha,-\alpha)}(h)\right\}
$$

Equivalently,

$$
\hat{\mathrm{k}}^{(\alpha,-\alpha)}(\cdot ; h)(\lambda)=\frac{1}{\lambda(\lambda+1)}\left\{\frac{\Gamma(\lambda+\alpha+1)}{\Gamma(\lambda+1) \Gamma(\alpha+1)}-\mathrm{P}_{\lambda}^{(\alpha,-\alpha)}(\mathrm{h})\right\}
$$

from (2.3).

When $\lambda=0, P_{0}^{(\alpha,-\alpha)}(x)=1$. This together with (2.7) yields

$$
\begin{aligned}
\hat{\mathrm{k}}^{(\alpha,-\alpha)}(\cdot ; \mathrm{h})(0) & =\frac{1}{2} \int_{-1}^{1}(1-\mathrm{x})^{\alpha}(1+\mathrm{x})^{-\alpha} \mathrm{k}(\mathrm{x} ; \mathrm{h}) \mathrm{dx} \\
& \left.=\frac{1}{2} \int_{\mathrm{h}}^{1}(1-\mathrm{x})^{\alpha}(1+\mathrm{x})^{-\alpha}\left\{\frac{1}{\alpha}\left[i \frac{1+\mathrm{x}}{1-\mathrm{x}}\right)^{\alpha}-\left(\frac{1+\mathrm{h}}{1-\mathrm{h}}\right)^{\alpha}\right]\right\} \mathrm{dx} \\
& =\frac{1}{2 \alpha}\left[1-\mathrm{h}-\left(\frac{1+\mathrm{h}}{1-\mathrm{h}}\right)^{\alpha} \int_{\mathrm{h}}^{1}\left(\frac{1-\mathrm{x}}{1+\mathrm{x}}\right)^{\alpha} \mathrm{dx}\right] .
\end{aligned}
$$

This completes the proof of Lemma 3.1 .

Since $\lambda^{\frac{1}{2}} \hat{k}^{(\alpha,-\alpha)}(\cdot, h)\left(\lambda-\frac{1}{2}\right) \varepsilon L^{l}\left(R^{+}\right)$and since $k(x ; h)$ is continuous on $(-1,1)$, it follows from $(2.8)$ and Lemma 3.1 that for $\lambda \neq 0$

$$
\begin{gathered}
k(x ; h)=4 \int_{0}^{\infty} \frac{1}{\lambda^{2}-\frac{1}{4}}\left\{\frac{\Gamma\left(\lambda+\frac{1}{2}\right)}{\Gamma\left(\lambda-\alpha+\frac{1}{2}\right) \Gamma(\alpha+1)}-\frac{\Gamma^{2}\left(\lambda+\frac{1}{2}\right) P_{\lambda-\frac{1}{2}}^{(\alpha,-\alpha)}(h)}{\Gamma\left(\lambda+\alpha+\frac{1}{2}\right) \Gamma\left(\lambda-\alpha+\frac{1}{2}\right)}\right\} . \\
\cdot P_{\lambda-\frac{1}{2}}^{(-\alpha, \alpha)}(-x) \lambda \sin \pi \lambda d \lambda .
\end{gathered}
$$

From (2.11) with $\mu=1, \sigma \geq 0, h \varepsilon(-1,1)$ and Lemma 3.1 , we have 


$$
\begin{aligned}
\hat{\mathrm{k}}^{(\alpha,-\alpha)}(\cdot, \mathrm{h})\left(\sigma-\frac{1}{2}\right)=\frac{1}{\sigma^{2}-\frac{1}{4}}\left[\frac{\Gamma\left(\sigma+\alpha+\frac{1}{2}\right)}{\Gamma\left(\sigma+\frac{1}{2}\right) \Gamma\left(\alpha+\frac{1}{2}\right)}-\mathrm{p}_{\sigma-\frac{1}{2}}^{(\alpha,-\alpha)}(\mathrm{h})\right] \\
=\frac{\Gamma\left(\sigma+\alpha+\frac{1}{2}\right) \hat{\mathrm{k}}^{(\alpha,-\alpha)}(\cdot ; \mathrm{h})(0) \sin \pi\left(\sigma-\frac{1}{2}\right)}{\pi\left(\sigma^{2}-\frac{1}{4}\right) \Gamma\left(\sigma+\frac{1}{2}\right) \Gamma(\alpha+1)}+ \\
+\sum_{n=1}^{\infty} \frac{(2 n+1) \Gamma(n+1) \Gamma\left(\sigma+\alpha+\frac{1}{2}\right) \sin \pi\left(\sigma-n-\frac{1}{2}\right)}{\pi\left(\sigma^{2}-\left(n+\frac{1}{2}\right){ }^{2}\right) \Gamma(n+\alpha+1) \Gamma\left(\sigma+\frac{1}{2}\right)} \frac{1}{n(n+1)} . \\
+\left[\frac{\Gamma(n+\alpha+1)}{\Gamma(\alpha+1) n !}-P_{n}^{(\alpha,-\alpha)}(x)\right]
\end{aligned}
$$

where $\hat{\mathrm{k}}^{(\alpha,-\alpha)}(\cdot ; \mathrm{h})(0)$ is as given in Lemma 3.1 . Replacing $\sigma$ by $\lambda+\frac{1}{2}$ in the above expression together with Lemma 3.1 and the uniqueness of the Jacobi transform imply

$$
\begin{aligned}
& \frac{1}{\lambda(\lambda+1)}\left[\frac{\Gamma(\lambda+\alpha+1)}{\Gamma(\alpha+1) \Gamma(\lambda+1)}-P_{\lambda}^{(\alpha,-\alpha)}(h)\right]=\frac{\Gamma(\lambda+\alpha+1) \sin \pi \lambda k^{\hat{n}}(\alpha,-\alpha)(x ; h)(0)}{\pi(\lambda)(\lambda+1) \Gamma(\alpha+1) \Gamma(\lambda+1)}+ \\
& \left.+\sum_{n=1}^{\infty} \frac{(2 n+1) \Gamma(n+1) \Gamma(\lambda+\alpha+1) \sin \pi(\lambda-n)}{\pi(\lambda-n)(\lambda+n+1) \Gamma(n+\alpha+1) \Gamma(\lambda+1)} \frac{1}{n(n+1)} \Gamma \frac{\Gamma(n+\alpha+1)}{\Gamma(\alpha+1) n !}-P_{n}^{(\alpha,-\alpha)}(x)\right]_{j} .
\end{aligned}
$$

Therefore,

$$
\begin{aligned}
& P_{\lambda}^{(\alpha,-\alpha)}(h)=\frac{\Gamma(\lambda+\alpha+1)}{\Gamma(\alpha+1) \Gamma(\lambda+1)}-\frac{\Gamma(\lambda+\alpha+1) \sin \pi \lambda \hat{k}^{(\alpha,-\alpha)}(\cdot, h)(0)}{\pi \Gamma(\alpha+1) \Gamma(\lambda-1)}- \\
& -\sum_{n=1}^{\infty} \frac{\lambda(\lambda+1)(2 n+1) \Gamma(\lambda+\alpha+1) \sin \pi(\lambda-n)}{\pi(\lambda-n)(\lambda+n+1) \Gamma(\lambda+1) n(n+1) \Gamma(\alpha+1)} \\
& +\sum_{n=1}^{\infty} \frac{\lambda(\lambda+1)(2 n+1) n ! \Gamma(\lambda+\alpha+1) \sin \pi(\lambda-n) P_{n}^{(\alpha,-\alpha)}(h)}{\pi(\lambda-n)(\lambda+n+1) \Gamma(n+\alpha+1) \Gamma(\lambda+1) n(n+1)}
\end{aligned}
$$

From (2.7) we now have

$$
\hat{P}_{\lambda}^{(\alpha,-\alpha)}(0)=\frac{1}{2} \int_{-1}^{1}(1-x)^{\alpha}(1+x)^{-\alpha} P_{0}^{(\alpha,-\alpha)}(x) P_{\lambda}^{(\alpha,-\alpha)}(x) d x
$$

which together with the above expression for $\hat{P}_{\lambda}^{(\alpha,-\alpha)}(h)$ yields

$$
\begin{aligned}
& \hat{\mathrm{P}}_{\lambda}^{(\alpha,-\alpha)}(0)=\frac{1}{2} \int_{-1}^{1}(1-\mathrm{x})^{\alpha}(1+\mathrm{x})^{-\alpha}\left[\frac{\Gamma(\lambda+\alpha+1)}{\Gamma(\alpha+1) \Gamma(\lambda+1)}\right. \\
& -\frac{\Gamma(\lambda+\alpha+1) \sin \pi \lambda \hat{k}^{(\alpha,-\alpha)}(x, h)(0)}{\pi \Gamma(\alpha+1) \Gamma(\lambda+1)} \\
& -\sum_{n=1}^{\infty} \frac{(2 n+1) \lambda(\lambda+1) \Gamma(\lambda+\alpha+1) \sin \pi(\lambda-n)}{\pi(\lambda-n)(\lambda+n+1) \Gamma(\lambda+1) n(n+1) \Gamma(\alpha+1)} \\
& +\sum_{n=1}^{\infty} \frac{(2 n+1) \lambda(\lambda+1) \Gamma(\lambda+\alpha+1) \sin \pi(\lambda-n) P_{\lambda}^{(\alpha,-\alpha)}(x)}{\pi(\lambda-n)(\lambda+n+1) \Gamma(n+\alpha+1) \Gamma(\lambda+1) n(2 n+1)}: d x
\end{aligned}
$$


Using Euler's formula [5]

$$
\int_{0}^{x}(x-t)^{\alpha} t^{\beta} d t=\frac{\Gamma(\alpha+1) \Gamma(\beta+1)}{\Gamma(\alpha+\beta-2)} x^{\alpha+\beta+1}, \alpha, \beta>-1
$$

with $\alpha+\beta=0, t=1+u$, we obtain

$$
\int_{-1}^{1}(1-x)^{\alpha}(1+x)^{-\alpha} d x=2 \Gamma(\alpha+1) \Gamma(1-\alpha) \text {. }
$$

This together with Lemma 3.1 yields

$$
\begin{aligned}
\hat{\mathrm{p}}(\alpha,-\alpha) & (0)=\frac{\Gamma(1-\alpha) \Gamma(\lambda+\alpha+1)}{\Gamma(\lambda+1)}- \\
& -\sum_{n=1}^{\infty} \frac{\lambda(\lambda+1)(2 n+1) \Gamma(1-\alpha) \Gamma(\lambda+\alpha+1) \sin \pi(\lambda-n)}{n(n+1) \pi(\lambda-n)(\lambda+n+1) \Gamma(\lambda+1)} \\
& -\frac{\Gamma(\lambda+\alpha+1) \sin \pi \lambda}{2 \pi \Gamma(\alpha+1) \Gamma(\lambda+1)} \int_{-1}^{1}(1-x)^{\alpha}(1+x)^{-\alpha} \frac{1}{2 \alpha}(1-x) d x \\
& +\frac{\Gamma(\lambda+\alpha+1) \sin \pi \lambda}{2 \pi \Gamma(\alpha+1) \Gamma(\lambda+1)} \int_{-1}^{1} \frac{(1-x)^{\alpha}(1+x)^{-\alpha}(1+x)^{\alpha}(1-x)^{-\alpha}}{2 \alpha} \int_{x}^{1}\left(\frac{1-t}{1+t}\right) \alpha d t d x \\
& +\sum_{n=1}^{\infty} \frac{\lambda(\lambda+1)(2 n+1) n ! \Gamma(\lambda+\alpha+1) \sin \pi(\lambda+n)}{\pi(\lambda-n)(\lambda+n+1) \Gamma(n+\alpha+1) \Gamma(\lambda+1) n(n+1)} . \\
& +\frac{1}{2} \int_{-1}^{1}(1-x)^{\alpha}(1+x)^{-\alpha} P_{n}^{(\alpha,-\alpha)}(x) d x .
\end{aligned}
$$

The last term in the above expression vanishes by the orthogonality of the Jacobi polynomials; that is,

$$
\begin{aligned}
& \int_{-1}^{1}(1-x)^{\alpha}(1+x)^{-\alpha} p_{n}^{(\alpha,-\alpha)}(x) d x= \\
& \quad=\int_{-1}^{1}(1-x)^{\alpha}(1+x)^{-\alpha} p_{0}^{(\alpha,-\alpha)}(x) P_{n}^{(\alpha,-\alpha)}(x) d x=0
\end{aligned}
$$

Moreover, using (3.5), the third term can be written

$$
\frac{1}{2} \int_{-1}^{1}(1-x)^{\alpha+1}(1+x)^{\alpha} d x=\Gamma(\alpha+2) \Gamma(1-\alpha) .
$$

Therefore,

$$
\begin{aligned}
& \hat{P_{\lambda}^{(\alpha,-\alpha)}}(0)=\frac{\Gamma(1-\alpha) \Gamma(\lambda+\alpha+1)}{\Gamma(\lambda+1)} \\
& -\sum_{n=1}^{\infty} \frac{\lambda(\lambda+1)(2 n+1) \Gamma(1-\alpha) \Gamma(\lambda+\alpha+1) \sin \pi(\lambda-n)}{n(n+1) \pi(\lambda-n)(\lambda+n+1) \Gamma(\lambda+1)}- \\
& \quad-\frac{\Gamma(\lambda+\alpha+1) \sin \pi \lambda}{2 \pi \Gamma(\lambda+1) \Gamma(\alpha+1) \alpha} \Gamma(\alpha+2) \Gamma(1-\alpha)+ \\
& +\frac{\Gamma(\lambda+\alpha+1) \sin \pi \lambda}{4 \pi \Gamma(\lambda+1) \Gamma(\alpha+1) \alpha} \int_{-1}^{1} \int_{x}^{1}\left(\frac{1-t}{1+t}\right)^{\alpha} d t d x .
\end{aligned}
$$


From $(2.6),(2.7)$ and the identity ${ }_{n}(\alpha, \beta)(-x)=(-1){ }^{n_{P}}(\beta, \alpha)(x)$, it follows that

$$
\hat{\mathrm{P}}_{\lambda}^{(\alpha,-\alpha)}(0)=\frac{\Gamma(\lambda+\alpha+1) \Gamma(1-\alpha) \sin \pi \lambda}{\pi \lambda(\lambda+1) \Gamma(\lambda+1)}, \lambda \neq 0, \lambda \geq-\frac{1}{2}
$$

Hence by the uniqueness of the Jacobi transform, we have from (3.6) and $(3.7)$,

$$
\begin{aligned}
1- & \sum_{n=1}^{\infty} \frac{\lambda(\lambda+1)(2 n+1) \sin \pi(\lambda-n)}{n(n+1) \pi(\lambda-n)(\lambda+n+1)}-\frac{\alpha+1}{2 \alpha} \frac{\sin \pi \lambda}{\pi}+ \\
& +\frac{\sin \pi \lambda}{4 \pi \alpha \Gamma(1+\alpha) \Gamma(1-\alpha)} \int_{-1}^{1} \int_{x}^{1}\left(\frac{1-t}{1+t}\right) \alpha d t=\frac{\sin \pi \lambda}{\pi \lambda(\lambda+1)}
\end{aligned}
$$

Now (3.4) can be expressed as

$$
\begin{aligned}
& \frac{\Gamma(\lambda+1) \lambda(\alpha+1)}{\Gamma(\lambda+\alpha+1)} P_{\lambda}^{(\alpha,-\alpha)}(x)= \\
& \quad=\lambda(\lambda+1) \sum_{n=1}^{\infty} \frac{(2 n+1) n ! \sin \pi(\lambda-n) P_{n}^{(\alpha,-\alpha)}(x)}{n(n+1)(\alpha+1) n^{\pi(\lambda-n)(\lambda+n+1)}}+ \\
& +\frac{\sin \pi \lambda}{\pi}\left[\frac{1}{2}+\frac{1}{\lambda(\lambda+1)}-\frac{\int_{-1}^{1} \int_{x}^{1}(1-t)^{\alpha}(1+t)-\alpha d t d x}{4 \alpha \Gamma(\alpha+1) \Gamma(1-\alpha)}+\right. \\
& +\frac{x}{2 \alpha}+\frac{1}{2 \alpha}\left(\frac{1+x}{1-x}\right)^{\alpha} \int_{x}^{1}\left(\frac{1-t}{1+t}\right)^{\alpha} d t !
\end{aligned}
$$

By interchanging the order of integration and by (3.5) we obtain

$$
\int_{-1}^{1} \int_{x}^{1}(1-t)^{\alpha}(1+t)^{-\alpha} d t d x=2 \Gamma(1+\alpha) \Gamma(2-\alpha)
$$

Thus,

$$
\begin{aligned}
& \frac{\Gamma(\lambda+1) \Gamma(\alpha+1)}{\Gamma(\lambda+\alpha+1)} \mathrm{P}_{\lambda}^{(\alpha,-\alpha)}(\mathrm{x})= \\
& =\lambda(\lambda+1) \sum_{n=1}^{\infty} \frac{(2 \mathrm{n}+1) \mathrm{n} !(-1)^{\mathrm{n}} \sin \pi \lambda \mathrm{P}_{\mathrm{n}}^{(\alpha,-\alpha)}(\mathrm{x})}{\mathrm{n}(\mathrm{n}+1)(\alpha+1) \mathrm{n}^{\pi(\lambda-n)(\lambda+n+1)}} \\
& +\frac{\sin \pi \lambda}{\pi} !_{1} 1+\frac{1}{\lambda(\lambda+1)}-\frac{1}{2 \alpha}+\frac{\mathrm{x}}{2 \alpha}+\frac{1}{2 \alpha}\left(\frac{1+\mathrm{x}}{1-\mathrm{x}}\right) \alpha \int_{\mathrm{x}}^{1}\left(\frac{1-t}{1+t}\right)^{\alpha} \mathrm{dt} !
\end{aligned}
$$

The series representation of the Jacobi function $P_{\lambda}^{(\alpha,-\alpha)}(x)$ will be completed once we obtain an equivalent expression for the integral.

$$
f(x ; \alpha)=\int_{x}^{1}\left(\frac{1-t}{1+t}\right)^{\alpha} d t
$$

Lemma 3.2. For $-\frac{1}{2}<\alpha<\frac{1}{2},(\alpha \neq 0)$, we have 
a) $f(x ; \alpha)=\frac{(1-x)^{\alpha+1}}{(1+x)^{\alpha}}\left(1-\frac{2 \alpha}{1+x} \sum_{n=1}^{\infty} \frac{(-1)^{n}}{\alpha+n+1}\left(\frac{1-x}{1+x}\right)^{n}\right), 0 \leq x<1$

b) $\quad f(x ; \alpha)=\frac{2 \pi \alpha}{\sin \pi \alpha}-\frac{(1-x)^{\alpha}}{(1+x)^{\alpha-1}}\left(1-\frac{2 \alpha}{1-x} \sum_{n=1}^{\infty} \frac{(-1)^{n}}{\alpha-n-1}\left(\frac{1+x}{1-x}\right)^{n}\right),-1<x<0$

Proof: a) Integration by parts yields the recursive relation

$$
f(x ; \alpha)=\frac{1}{\alpha+1} \frac{(1-x)^{\alpha+1}}{(1+x)^{\alpha}}-\frac{\alpha}{1+1} f(x ; \alpha+1) .
$$

By employing this relation and after simplification, we obtain

$$
f(x ; \alpha)=\frac{(1-x)^{\alpha+1}}{(1+x)^{\alpha}}\left(1-\frac{2 \alpha}{1+} \sum_{n=0}^{\infty} \frac{(-1)^{n}}{n+\alpha+1}\left(\frac{1-x}{1+x}\right)^{n}\right)
$$

The series converges for all $x$ such that $\left|\frac{1-x}{1+x}\right|<1$; that is, if $0: x<1$. When $x=0$,

$$
f(0 ; \alpha)=1-2 \alpha \sum_{n=0}^{\infty} \frac{(-1)^{n}}{n+\alpha+1} .
$$

b) We rewrite $f(x ;)$ as

$$
f(x ; \alpha)=\int_{x}^{0}\left(\frac{1-t}{1+t}\right)^{\alpha} d t+\int_{0}^{1}\left(\frac{1-t}{1+t}\right)^{\alpha} d t=J(x ; \alpha)+f(0, \alpha) \text {, say. }
$$

By introducing

$$
J *(x ; \alpha)=\int_{-1}^{x}\left(\frac{1-t}{1+t}\right) \alpha d t
$$

$J(x ; \alpha)$ can be written as

$$
J(x ; \alpha)=J^{\star}(0 ; \alpha)-J^{\star}(x ; \alpha) .
$$

Upon an integration by parts, we obtain

$$
J \star(x ; \alpha)=\frac{1}{1-\alpha} \frac{(1-x)^{\alpha}}{(1+x)^{\alpha-1}}+\frac{\alpha}{1-\alpha} J *(x ; \alpha-1)
$$

Repeating the above formula, recursively, results in the series.

$$
J^{*}(x ; \alpha)=\frac{(1-x)^{\alpha}}{(1+x)^{\alpha-1}}\left(1-\frac{2 \alpha}{1-x} \sum_{n=0}^{\infty} \frac{(-1)^{n}}{\alpha-n-1}\left(\frac{1+x}{1-x}\right)^{n}\right)
$$

which converges for all $x$ such that $\left|\frac{1+x}{1-x}\right|<1$; that is, for $-1<x<0$. When $x=0$,

$$
J *(0 ; \alpha)=1-2 \alpha \sum_{n=0}^{\infty} \frac{(-1)^{n}}{\alpha-n-1} .
$$


Thus,

$$
\begin{aligned}
f(x ; \alpha) & =J^{\star}(0, \alpha)+f(0, \alpha)-J^{*}(x ; \alpha) \\
& =\frac{2 \pi \alpha}{\sin \pi \alpha}-\frac{(1-x)^{\alpha}}{(1+x)^{\alpha-1}}\left(1-\frac{2 \alpha}{1-x} \sum_{n=0}^{\infty} \frac{(-1)^{n}}{\alpha-n-1}\left(\frac{1+x}{1-x}\right)^{n}\right)
\end{aligned}
$$

which completes the verification of Lemma 3.2 .

From (3.8) and Lemma 3.2, the representation of the Jacobi function $P_{\lambda}^{(\alpha,-\alpha)}(x)$ will follow. In particular, for $\lambda \geq-\frac{1}{2}(\lambda \neq 0)$

$$
\begin{aligned}
& \mathrm{P}_{\lambda}^{(\alpha,-\alpha)}(\mathrm{x})=\frac{\Gamma(\lambda+\alpha+1)}{\Gamma(\lambda+1) \Gamma(\alpha+1)} \frac{\sin \pi \lambda}{\pi}\{\lambda(\lambda+1) \\
& -\sum_{n=1}^{\infty} \frac{(2 \mathrm{n}+1) \mathrm{n} !(-1)^{\mathrm{n}} \mathrm{P}_{\mathrm{n}}(\alpha,-\alpha)(\mathrm{x})}{\mathrm{n}(\mathrm{n}+1)(\alpha+1){ }_{\mathrm{n}}(\lambda-\mathrm{n})(\lambda+\mathrm{n}+1)}+1+\frac{1}{\lambda(\lambda+1)}- \\
& \left.-\sum_{n=0}^{\infty} \frac{(-1)^{\mathrm{n}}}{\mathrm{n}+\alpha+1}\left(\frac{1-\mathrm{x}}{1+\mathrm{x}}\right)^{\mathrm{n}+1}\right\}, 0 \leq \mathrm{x}<1 ;
\end{aligned}
$$

and

$$
\begin{aligned}
& \mathrm{P}_{\lambda}^{(\alpha,-\alpha)}(\mathrm{x})=\frac{\Gamma(\lambda+\alpha+1)}{\Gamma(\lambda+1) \Gamma(\alpha+1)} \frac{\sin \pi \lambda}{\pi}\{\lambda(\lambda+1) \cdot \\
& -\sum_{n=1}^{\infty} \frac{(2 n+1) n !(-1)^{n} \mathrm{P}_{n}(\alpha,-\alpha)(x)}{\mathrm{n}(\mathrm{n}+1)(\alpha+1)}{ }_{\mathrm{n}}^{(\lambda-n)(\lambda+n+1)}+1+\frac{1}{\lambda(\lambda+1)}-\frac{1}{\alpha}+ \\
& \left.+\frac{(1+x)^{\alpha}}{(1-x)^{\alpha}} \frac{\pi}{\sin \pi \alpha}+\sum_{n=0}^{\infty} \frac{(-1)^{n}}{\alpha-n-1}\left(\frac{1+x}{1-x}\right)^{n+1}\right\},-1<x<0 .
\end{aligned}
$$

The above representations will hold for $\lambda=0$ provided that $\frac{\sin \pi \lambda}{\pi \lambda}$ is interpreted to be equal to 1 for $\lambda=0$. When $\alpha=0$, the formula reduces to that for Legendre functions derived in [3], provided that $-\frac{1}{\alpha}+\frac{(1+x)^{\alpha}}{(1-x)^{\alpha}} \frac{\pi}{\sin \pi \alpha}$ is given its limiting value of 0 as $\alpha \rightarrow 0$.

\section{REFERENCES}

[1] Deeba, E.Y. and E.L. Koh, The continuous Jacobi transform, Internat. J. Math and Math. Sci. Vol.6, No. 1 (1983), 145-160.

[2] Deeba, E.Y. and E.L. Koh, The second continuous Jacobi transform, Internat. J. Math and Math Sci. (to appear).

[3] Butzer, P.L., R.L. Stens and M. Wehrens, The continuous Legendre transform, its inverse transform and applications, Internat. J. Math and Math Sc. Vol. 3, No. 1 (1980), 47-67. 
[4] Debnath, L., On Jacobi transform, Bull. Calc. Math. Soc., Vol. 55 (1963), 113-120.

[5] Luke, Y.L., The Special Functions and Their Applications, Vol. I, Academic Press, New York, 1969.

[6] Erdelyi, A., W. Magnus, F. Oberhettinger and F.G. Tricomi, Higher Transcendental Functions, Vol. 1, McGraw Hill, New York, 1953. 


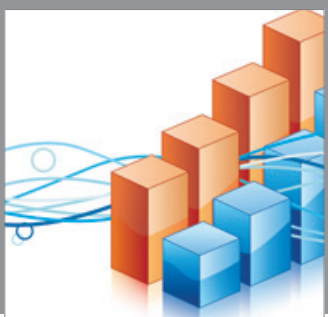

Advances in

Operations Research

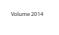

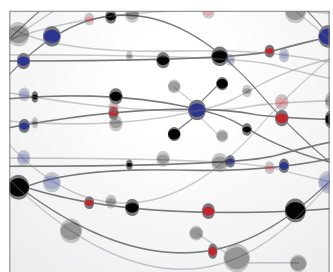

\section{The Scientific} World Journal
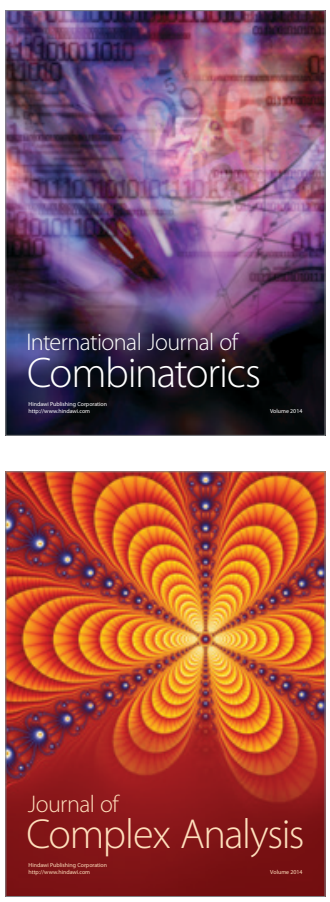

International Journal of

Mathematics and

Mathematical

Sciences
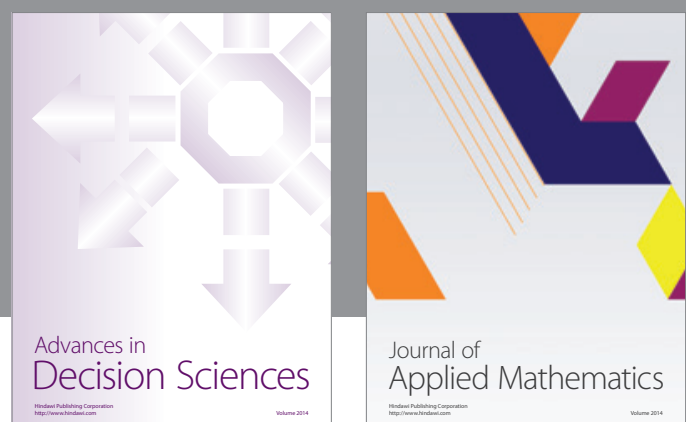

Journal of

Applied Mathematics
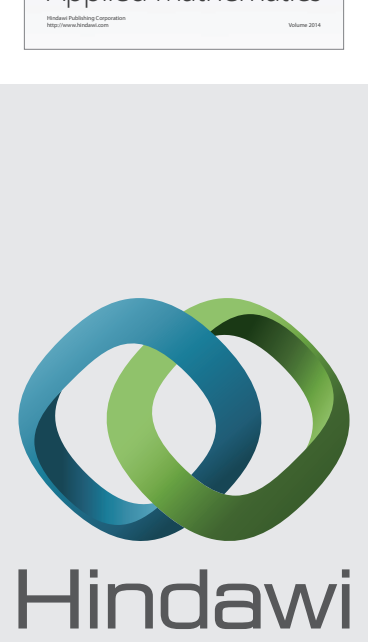

Submit your manuscripts at http://www.hindawi.com
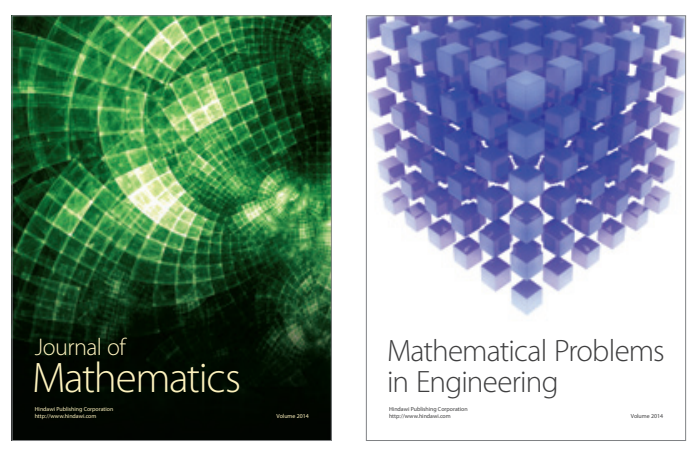

Mathematical Problems in Engineering
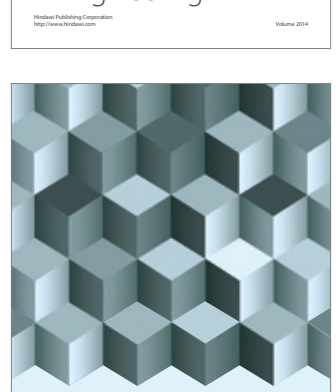

Journal of

Function Spaces
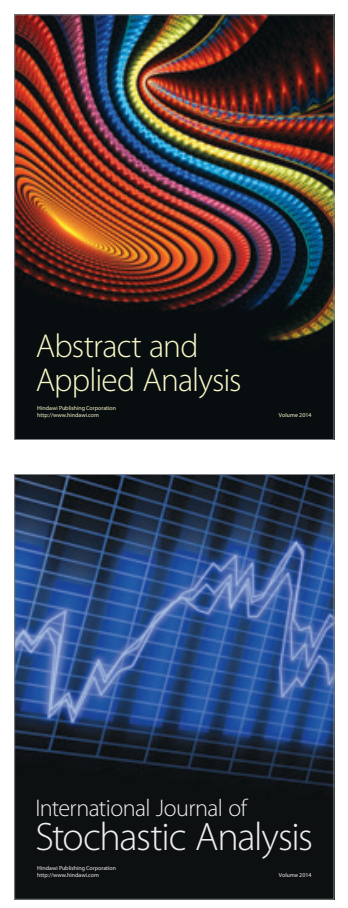

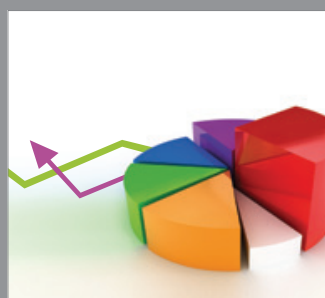

ournal of

Probability and Statistics

Promensencen
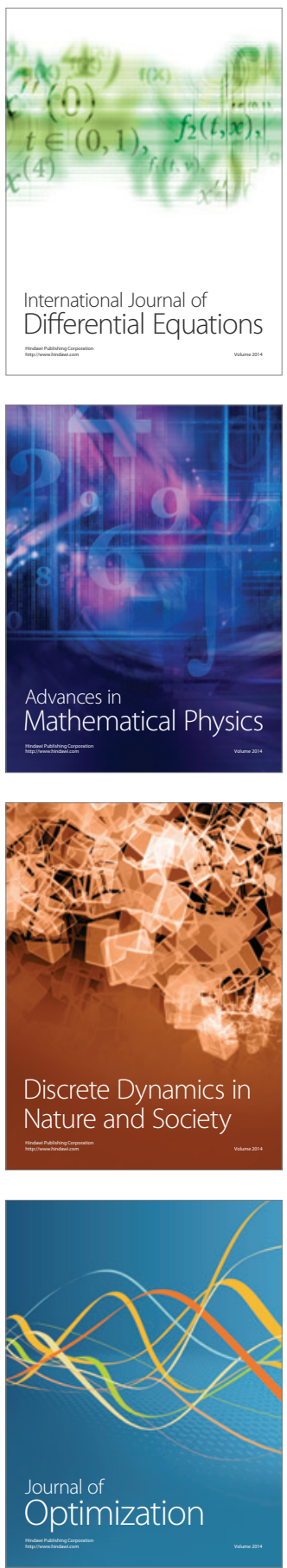